TITLE:

\title{
Femtosecond upconverted photocurrent spectroscopy of InAs quantum nanostructures
}

\section{$\operatorname{AUTHOR}(\mathrm{S}):$}

Yamada, Yasuhiro; Tex, David M.; Kamiya, Itaru; Kanemitsu, Yoshihiko

\section{CITATION:}

Yamada, Yasuhiro ... [et al]. Femtosecond upconverted photocurrent spectroscopy of InAs quantum nanostructures. Applied Physics Letters 2015, 107 (01): 013905.

\section{ISSUE DATE:}

2015-07-08

URL:

http://hdl.handle.net/2433/210115

\section{RIGHT:}

Copyright 2015 AlP Publishing. This article may be downloaded for personal use only. Any other use requires prior permission of the author and AIP Publishing. The following article may be found at

http://scitation.aip.org/content/aip/journal/apl/107/1/10.1063/1.49265 69 


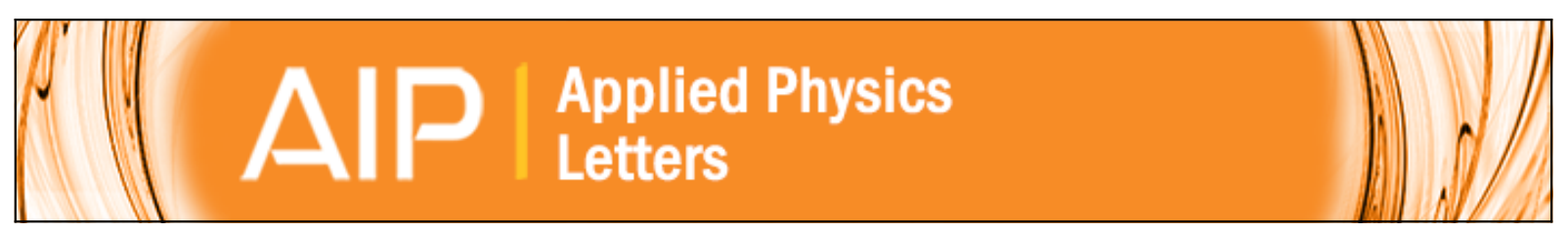

\section{Femtosecond upconverted photocurrent spectroscopy of InAs quantum nanostructures}

Yasuhiro Yamada, David M. Tex, Itaru Kamiya, and Yoshihiko Kanemitsu

Citation: Applied Physics Letters 107, 013905 (2015); doi: 10.1063/1.4926569

View online: http://dx.doi.org/10.1063/1.4926569

View Table of Contents: http://scitation.aip.org/content/aip/journal/apl/107/1?ver=pdfcov

Published by the AIP Publishing

\section{Articles you may be interested in}

Competitive carrier interactions influencing the emission dynamics of GaAsSb-capped InAs quantum dots Appl. Phys. Lett. 101, 231109 (2012); 10.1063/1.4769431

Photocurrent spectroscopy of intersubband transitions in GalnAsN/(Al)GaAs asymmetric quantum well infrared photodetectors

J. Appl. Phys. 112, 084502 (2012); 10.1063/1.4754573

Properties of the SiO2- and SiNx-capped GaAs(100) surfaces of GalnAsN/GaAs quantum-well heterostructures studied by photoelectron spectroscopy and photoluminescence

Appl. Phys. Lett. 99, 102105 (2011); 10.1063/1.3634046

Structural and optical properties of vertically stacked triple InAs dot-in-well structure

J. Appl. Phys. 103, 096107 (2008); 10.1063/1.2921266

Carrier relaxation in InGaN/GaN quantum wells with nanometer-scale cluster structures

Appl. Phys. Lett. 85, 1371 (2004); 10.1063/1.1784033

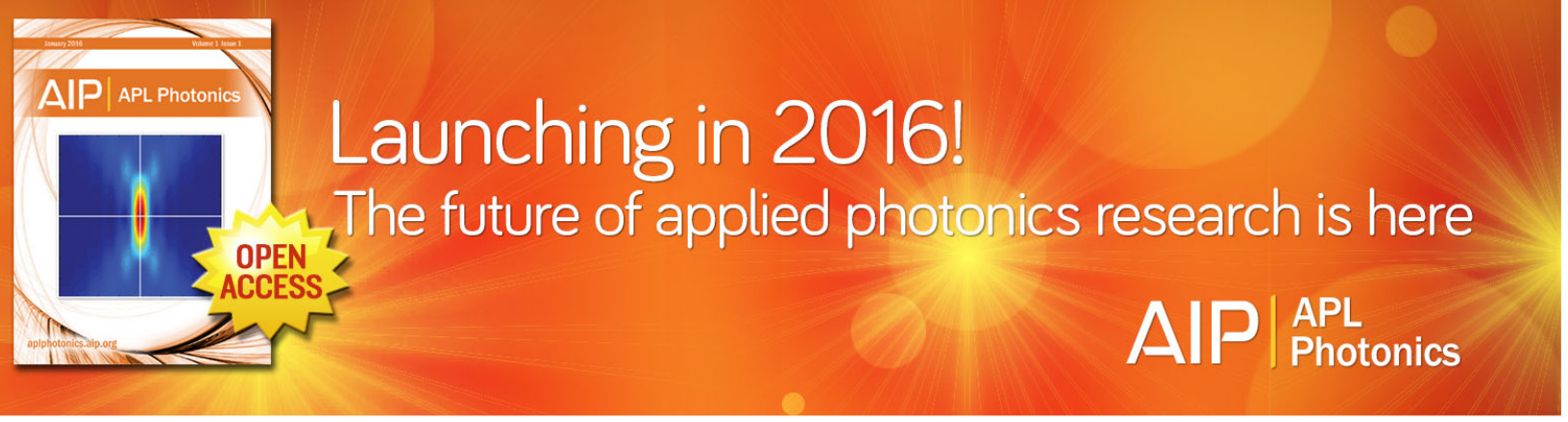




\title{
Femtosecond upconverted photocurrent spectroscopy of InAs quantum nanostructures
}

\author{
Yasuhiro Yamada, ${ }^{1}$ David M. Tex, ${ }^{1,2}$ Itaru Kamiya, ${ }^{3}$ and Yoshihiko Kanemitsu ${ }^{1,2, a)}$ \\ ${ }^{1}$ Institute for Chemical Research, Kyoto University, Uji, Kyoto 611-0011, Japan \\ ${ }^{2}$ Japan Science and Technology Agency, CREST, Kyoto University, Uji, Kyoto 611-0011, Japan \\ ${ }^{3}$ Toyota Technological Institute, Nagoya, Aichi 468-8511, Japan
}

(Received 8 January 2015; accepted 28 June 2015; published online 8 July 2015)

\begin{abstract}
The carrier upconversion dynamics in InAs quantum nanostructures are studied for intermediateband solar-cell applications via ultrafast photoluminescence and photocurrent (PC) spectroscopy based on femtosecond excitation correlation (FEC) techniques. Strong upconverted PC-FEC signals are observed under resonant excitation of quantum well islands (QWIs), which are a few monolayer-thick InAs quantum nanostructures. The PC-FEC signal typically decays within a few hundred picoseconds at room temperature, which corresponds to the carrier lifetime in QWIs. The photoexcited electron and hole lifetimes in InAs QWIs are evaluated as functions of temperature and laser fluence. Our results provide solid evidence for electron-hole-hole Auger process, dominating the carrier upconversion in InAs QWIs at room temperature. (C) 2015 AIP Publishing LLC.

[http://dx.doi.org/10.1063/1.4926569]
\end{abstract}

The quantum confinement of electrons in nanostructures enhances dynamical electron-electron interactions, thereby leading to many-body processes such as quantized Auger recombination (AR) and carrier multiplication $(\mathrm{CM}){ }^{1-6} \mathrm{In}$ AR process, the recombination energy of an electron-hole (e-h) pair is transferred to a third electron or hole which results in a carrier with large kinetic energy, i.e., a so-called hot carrier. ${ }^{5,6}$ In $\mathrm{CM}$ process, a single high-energy photon creates two or more e-h pairs. ${ }^{2-6}$ Unique solar-cell concepts based on these AR and CM processes in nanostructures have attracted considerable attention because of the high theoretical conversion-efficiency limit beyond that of conventional single $\mathrm{p}-\mathrm{n}$ junction solar cells. For instance, confined photocarriers in semiconductor nanostructures embedded in bulk crystals can be efficiently extracted above the potential barrier via upconversion through multi-carrier interactions, which can assist in realizing efficient intermediate-band solar cells (IBSCs). ${ }^{7-10}$

InAs quantum nanostructures such as quantum dots (QDs) have tremendous potential for future SC applications. In the case of IBSCs, low-energy infrared photons can be absorbed by InAs nanostructures embedded in GaAs, and two-step excitation of charge carriers to higher energy levels enables enhanced photocurrent (PC). ${ }^{9-12}$ While the efficiency of this upconversion process is a core requirement for IBSCs, the currently achieved efficiencies are far from that needed for practical SC applications. We found that disk-like InAs nanostructures embedded in $\mathrm{AlGaAs}$, which are referred to as quantum well islands (QWIs), exhibit efficient upconverted PC, and that the large efficiency of the PC cannot be explained by conventional thermal and two-step photoabsorption processes. ${ }^{9,10}$ Although it has been suggested that the enhanced PC is caused by upconversion of photocarriers through the AR process, no direct evidence has been presented experimentally, ${ }^{8,9}$ and the detailed mechanism

${ }^{a)}$ E-mail: kanemitu@scl.kyoto-u.ac.jp remains unclear. It is necessary to clarify the physical mechanism of the efficient carrier upconversion in QWIs to realize efficient IBSCs. In addition, an evaluation of electron and hole lifetimes to gain a thorough understanding of the upconversion process in InAs nanostructures can aid in arriving at the design principles required for the development of highly efficient SCs based on quantum nanostructures. ${ }^{12,13}$

Time-resolved spectroscopy is one of the most important techniques to investigate the dynamical behaviors of photocarriers that determine the photovoltaic characteristics of SCs. ${ }^{14,15}$ Popular techniques based on femtosecond photoluminescence (PL) and transient absorption (TA) have been used to investigate the ultrafast response of photocarriers in various kinds of SC materials including nanostructures. ${ }^{16,17}$ Further, time-resolved PC measurement is a powerful tool for analyzing carrier dynamics. ${ }^{18-21}$ In particular, the PC measurement directly probes the charge carriers extracted from the nanostructures in IBSCs, and thus, we can obtain essential information about the carrier generation and extraction processes. However, the ultrafast response of PC measurements has not been fully investigated thus far when compared with PL and TA spectroscopies because the fabrication of complex device structures such as the Auston switch is necessary for PC measurements. ${ }^{22,23}$

In this letter, we investigated the ultrafast upconverted PC dynamics in InAs quantum nanostructures by femtosecond excitation correlation (FEC) spectroscopy to unveil the carrier upconversion processes. We observed efficient upconverted PC and PC-FEC signals upon excitation of shallow InAs QWIs. The room-temperature PC-FEC decay time was typically $\sim 100 \mathrm{ps}$, depending on the excitation laser fluence. We evaluated the photoexcited electron and hole lifetimes based on PL- and PC-FEC measurements as functions of temperature and laser fluence. The strong dependence of the carrier lifetime on laser fluence provides solid evidence that the three-carrier AR process dominates the carrier upconversion process in InAs QWIs at room temperature. 
The sample was prepared by molecular beam epitaxy under conditions similar to those described in our previous publication. ${ }^{9}$ A nominally undoped structure was grown on top of a semi-insulating GaAs (001) substrate and a GaAs buffer. The sample contains an AlGaAs layer with an InAs layer nominally two monolayer (ML) thick. It was confirmed that the PC signals from this layer are dominant and therefore this layer will be discussed in detail. It is known that one ML wetting layers (WLs) and QDs are self-organized in the thin InAs layer. In addition, disk-like InAs nanostructures (QWIs) that are a few MLs high are also formed. ${ }^{8,9,24}$ The sample structure and the energy diagram are schematically illustrated in Fig. 1(a). ${ }^{25}$ For this sample, we have determined the resonance energy of InAs QD, 2 ML-QWI, 3 MLQWI, and WL in AlGaAs barrier by means of conventional PL and PC spectroscopy. ${ }^{8-10}$ For instance, the resonance energy for 2-ML QWIs at room temperature is $1.37 \mathrm{eV}$, and QD resonance energy widely ranges below $1.25 \mathrm{eV} .^{9}$ (For more details of this sample, see Ref. 25.)

For PC measurements, an open-rectangular-shaped gold electrode was evaporated on the GaAs top layer and excitation laser beam was focused on the sample surface surrounded by the electrode [see Fig. 1(b)]. Indium metal was attached on the GaAs substrate as a bottom electrode. For ultrafast time-resolved PC measurements, we applied the FEC technique, which is often used in PL spectroscopy. ${ }^{26,27}$ A positive bias voltage of $0.2 \mathrm{~V}$ was applied to the indium electrode to gain sufficient PC. We confirmed that the FEC profile is independent of the bias voltage. The excitation laser source was a wavelength-tunable femtosecond laser system (pulse duration: $250 \mathrm{fs}$ and repetition rate: $200 \mathrm{kHz}$ ). As shown in Fig. 1(b), two femtosecond pulses, modulated at different frequencies $\left(\mathrm{f}_{1}=391 \mathrm{~Hz}\right.$ and $\mathrm{f}_{2}=326 \mathrm{~Hz}$ ), were focused on the sample surface. The FEC signal was detected by a lock-in amplifier with a differential frequency of $\mid f_{1}-$ $\mathrm{f}_{2} \mid$ as a function of the delay time between the two pulses, $\tau$. The FEC signal arises due to the temporal overlap of the PC induced by the first and second pulses via a nonlinear response, as schematically described in Fig. 1(c). For a carrier lifetime of $\tau_{\mathrm{c}} \ll \tau$, there is no temporal overlap between the carriers induced by the first and second pulses, and thus, the FEC signal intensity is zero. Conversely, when $\tau_{\mathrm{c}} \sim \tau$, a temporal overlap between carriers generated by the first and second pulses occurs, and this overlap results in a variation in the total PC intensity because of the nonlinear interaction between the carriers. If we assume that the PC intensity is proportional to the carrier density and shows weak nonlinearity on excitation intensity, the FEC signal intensity is approximately written as ${ }^{25}$

$$
\mathrm{FEC} \propto n\left(N_{1}, t\right) N_{2} . \quad\left(\text { for } N_{1} \ll N_{2}\right) .
$$

Here, $n(N, t)$ represents the carrier density at time $t$ after the initial carrier density $N$ induced by photoexcitation at time 0 . $N_{1}$ and $N_{2}$ denote the initial photocarrier densities generated by the first and second pulses. Note that the FEC signal intensity is zero when $n(N, t)$ depends linearly on $N$, and positive (negative) FEC signal corresponds to sublinear (superlinear) excitation-intensity dependence, as schematically shown in the inset of Fig. 1(c). According to Eq. (1), the FEC profile corresponds to the population dynamics of carriers that contribute to the PC.

For the temperature dependence of the PC-FEC profile, the excitation photon energy was tuned to the QWI resonance energy at each temperature. PL signals were detected by an InGaAs photodiode, and PL-FEC signals were obtained in a similar manner with PC-FEC. The time-resolutions of PCand PL-FEC measurements were approximately 250 fs, which is determined by the duration of the excitation laser pulse.

Figure 2(a) shows the PC-FEC profiles at room temperature under different excitation photon energies. The PC-FEC profiles are normalized and offset for clarity. The first and second pulse fluences were 3.2 and $20 \mu \mathrm{J} / \mathrm{cm}^{2}$, respectively. (a)
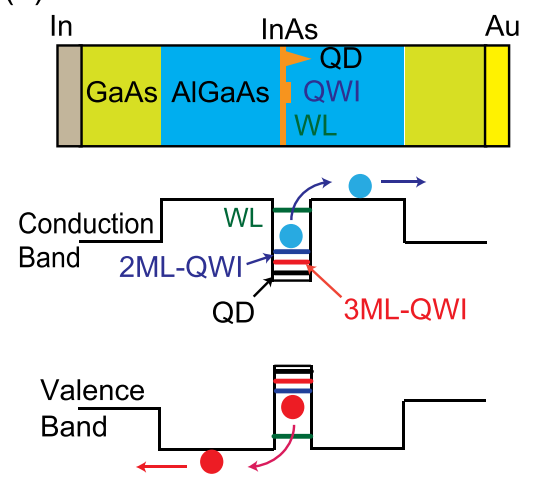

(b)

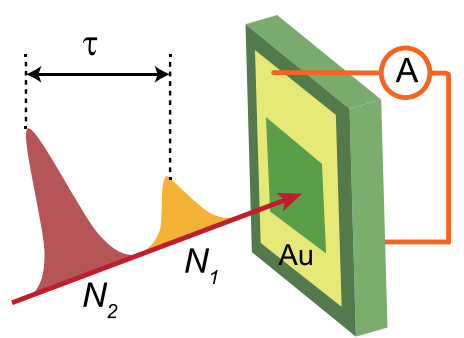

(c)
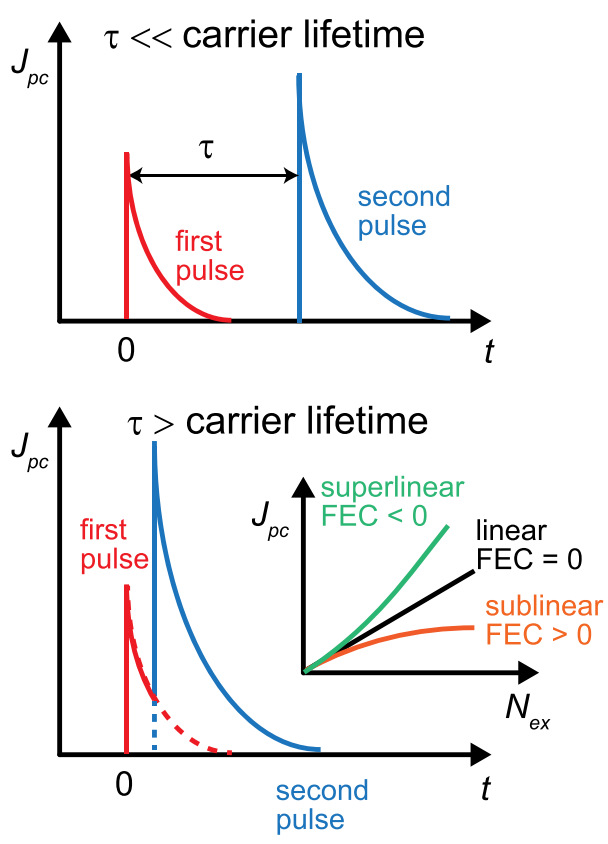

FIG. 1. (a) Schematic illustration of sample structure and energy diagram. Electrons and holes photoexcited in QWIs are upconverted to AlGaAs barrier layers and contribute to PC. (b) Experimental setup for PC-FEC measurements. (c) Schematic of FEC signal interpretation. Decay dynamics of carrier density under excitation by first and second pulses are shown for $\tau \ll$ carrier lifetime and $\tau>$ carrier lifetime. The inset shows excitation-fluence dependence of PC. The sign of FEC signal is determined whether PC superlinearly or sublinearly depends on excitation-fluence according to Eq. (1). 

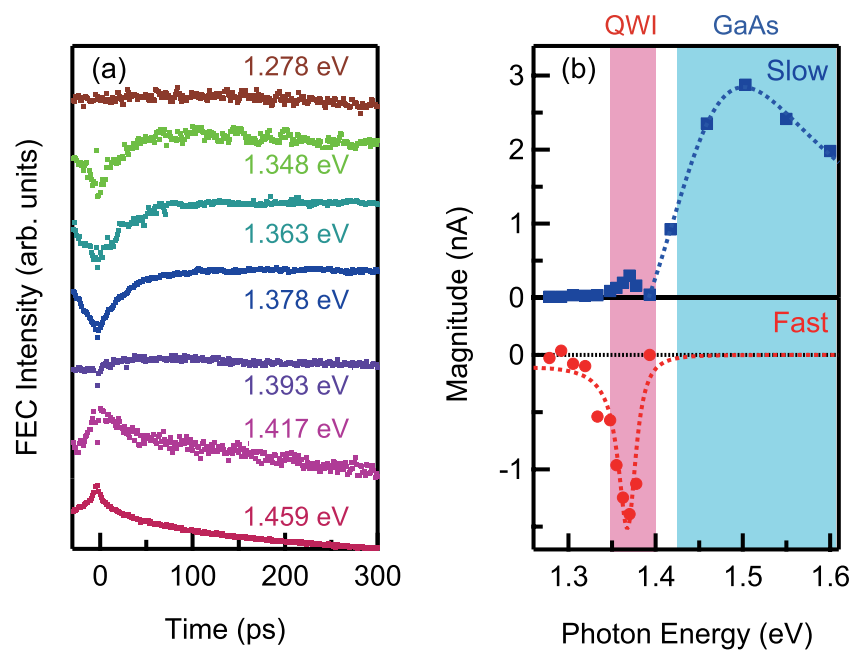

FIG. 2. (a) Excitation photon energy dependence of PC-FEC profiles. (b) Magnitudes of slow and fast components as a function of excitation photon energy.

Above the excitation energy of the GaAs substrate (around $1.42 \mathrm{eV}$ ), the FEC signal exhibits a positive slow-decay component. The decay profile is non-exponential and well reproduced by a double exponential function, $I_{\mathrm{s} 1} \exp (-t /$ $\left.\tau_{\mathrm{s} 1}\right)+\mathrm{I}_{\mathrm{s} 2} \exp \left(-\mathrm{t} / \tau_{\mathrm{s} 2}\right)$. The mean lifetime, $\left(\mathrm{I}_{\mathrm{s} 1} \tau_{\mathrm{s} 1}+\mathrm{I}_{\mathrm{s} 2} \tau_{\mathrm{s} 2}\right) /$ $\left(\mathrm{I}_{\mathrm{s} 1}+\mathrm{I}_{\mathrm{s} 2}\right)$, was $\sim 700$ ps and almost independent of the excitation photon energy above $1.42 \mathrm{eV}$. The positive FEC signal indicates a sublinear excitation-fluence dependence of the PC. We observed strong PC and PC-FEC signals under excitation at $1.37 \mathrm{eV}$, which correspond to the $2 \mathrm{ML}-\mathrm{QWI}$ resonance. ${ }^{8,9}$ Here, we remark that we observed considerably smaller PC and PC-FEC signals under resonant excitation of QDs $(>1.25 \mathrm{eV})$ compared with those of QWIs, thereby indicating that efficient upconversion occurs in QWIs. Therefore, we hereafter focus on the QWI.

Under QWI resonant excitation, a fast and negative decay component appears. The fast decay time, $\tau_{\mathrm{f}}$, was on the timescale of several tens of picoseconds, depending on the laser fluence; this is discussed later. We fitted the experimental data by a triple exponential function, $\mathrm{I}_{\mathrm{s} 1} \exp (-\mathrm{t} /$ $\left.\tau_{\mathrm{s} 1}\right)+\mathrm{I}_{\mathrm{s} 2} \exp \left(-\mathrm{t} / \tau_{\mathrm{s} 2}\right)+\mathrm{I}_{\mathrm{f}} \exp \left(-\mathrm{t} / \tau_{\mathrm{f}}\right)$ for $\mathrm{t}>0$, where $\mathrm{I}_{\mathrm{f}}<0$, $\mathrm{I}_{\mathrm{s} 1}>0$, and $\mathrm{I}_{\mathrm{s} 2}>0$. The magnitudes of the slow $\left(\mathrm{I}_{\mathrm{s} 1}+\mathrm{I}_{\mathrm{s} 2}\right)$ and fast $\left(\mathrm{I}_{\mathrm{f}}\right)$ components are shown in Fig. 2(b). The positive slow-decay component is mainly observed above the bandgap energy of GaAs $(1.42 \mathrm{eV})$. Thus, the slow-decay time corresponds to the photocarrier lifetime in GaAs. The fast-decay component has a peak at $1.37 \mathrm{eV}$, which corresponds to the QWI resonance energy, ${ }^{9}$ thereby indicating that the fast-decay time is the photocarrier lifetime in QWIs. The negative FEC intensity appearing at the excitation energy of the QWIs indicates a superlinear excitationfluence dependence of the PC, which can be caused by the upconversion of carriers to GaAs through the AR process.

Since the AR rate is sensitive to the number of photoexcited carriers, ${ }^{1,5,6}$ we studied the excitation-fluence dependence of the PC-FEC profiles under resonant excitation of QWIs, as shown in Fig. 3(a). The PC-FEC profiles are normalized and offset for clarity. Here, the excitation photon energy was tuned to $1.37 \mathrm{eV}$, which is the resonance energy of the QWI. The second pulse fluence was fixed at $20 \mu \mathrm{J} / \mathrm{cm}^{2}$. The
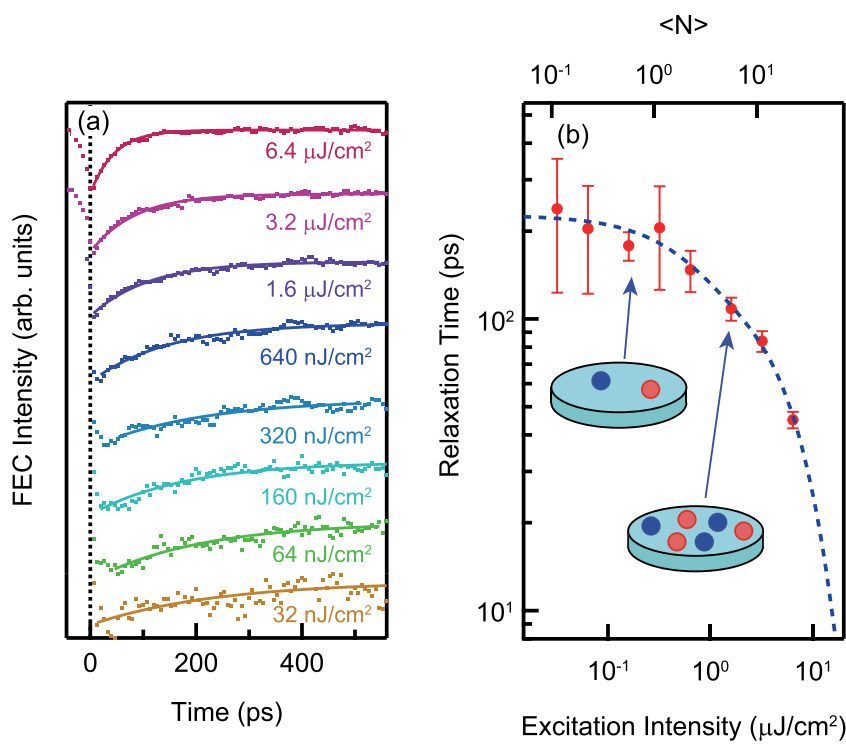

FIG. 3. (a) Excitation-laser-fluence dependence of PC-FEC profiles. (b) Relaxation time as a function of excitation laser fluence. The estimated carrier density per QWI is represented along the top $\mathrm{x}$-axis.

time constant of the fast-decay component is plotted as a function of the first pulse fluence in Fig. 3(b). Here, we roughly estimated the mean number of $\mathrm{e}-\mathrm{h}$ pairs photogenerated in a QWI, $\langle N\rangle$. We used a QWI with a diameter of $100 \mathrm{~nm}$, and we assumed that $1 \%$ of the excitation light was absorbed (assuming almost bulk-like absorption). Below a laser fluence of $500 \mathrm{~nJ} / \mathrm{cm}^{2}$, the relaxation time is nearly constant at $\sim 200$ ps. This means that multi-carrier interactions are negligible and indicates that the relaxation time at low excitation densities represents the intrinsic carrier lifetime in QWIs. This is consistent with the estimated number of $\langle\mathrm{N}\rangle<1$. Above 500 $\mathrm{nJ} / \mathrm{cm}^{2}$, the relaxation time decreases with increasing excitation intensity. Under high-fluence excitation, a large number of electrons and holes are photogenerated in a QWI, and the AR process dominates the carrier recombination dynamics. Such excitation-density dependent carrier dynamics cannot be accounted for by two-step two-photon absorption process because two-step two-photon absorption process occurs within the pulse duration and never affect the carrier dynamics after pulse excitation. It should be noted that the assessment of the carrier lifetime in QWIs plays a key role for the design of devices based on InAs nanostructures.

The PC can arise from both electrons and holes. Thus, it is necessary to verify whether the observed lifetime corresponds to that of electrons or holes. For this purpose, we compared the PC-FEC results with the PL-FEC results. The PL from the QWI was observed only at low temperatures below $100 \mathrm{~K}$ because of thermal quenching. The excitation photon energy was $1.49 \mathrm{eV}$, which is close to the QWI resonance energy $(1.46 \mathrm{eV})$ at low temperatures below $100 \mathrm{~K}$. The PL was monitored at $1.43 \mathrm{eV}$. The normalized PL- and PC-FEC profiles at $12 \mathrm{~K}$ are plotted in Fig. 4(a). The excitation fluences were 5 and $60 \mu \mathrm{J} / \mathrm{cm}^{2}$ for the first and second pulses, respectively. The PL-FEC profile shows a single decay component with a lifetime of $\sim 100$ ps. On the other hand, the PC-FEC profile exhibits two components. The fast $\mathrm{PC}$ component is similar to the PL decay profile and the 
(a)

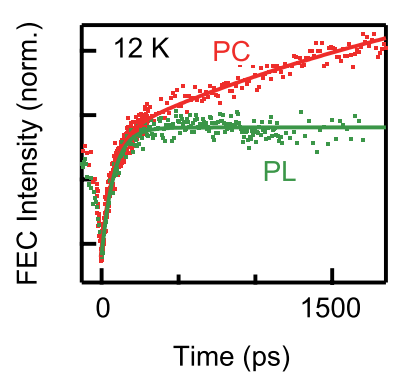

(c)
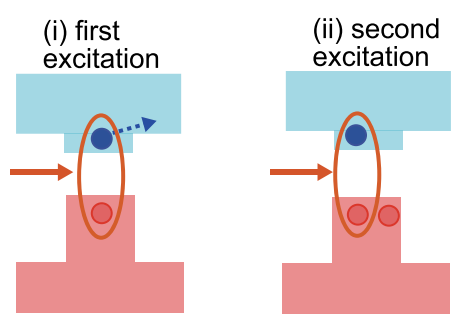

(b)

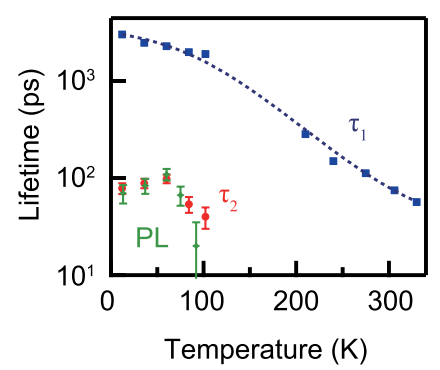

FIG. 4. (a) PC- and PL-FEC profiles at $12 \mathrm{~K}$. (b) Temperature dependence of the decay times $\left(\tau_{1}, \tau_{2}\right.$, and PL lifetime). (c) Schematics of roomtemperature relaxation and upconversion processes involving photoexcited electrons and holes. (i) An electron photoexcited by the first pulse is thermally upconverted to the barrier and a photoexcited hole is left behind in the QWI. (ii) Via the second-pulse excitation, an electron-hole-hole state is realized in the QWI. (iii) The hole is upconverted to the barrier via the three-carrier Auger process.

slow decay is of the order of nanoseconds. Note that the directions of the left and right axes in Fig. 4(a) are opposite, i.e., the PL-FEC signal is plotted upside down. Hence, PLFEC has a positive decaying component, while PC-FEC shows a negative component. This is because AR process causes a reduction of the PL efficiency, and therefore, PL shows the sublinear excitation-fluence dependence, which results in the positive FEC signals.

The fast $\left(\tau_{1}\right)$ and slow $\left(\tau_{2}\right)$ PC lifetimes and the PL lifetime are plotted as a function of temperature in Fig. 4(b). The fast PC lifetime $\tau_{1}$ increases monotonically with decreasing temperature and reaches a value of $3 \mathrm{~ns}$ at $12 \mathrm{~K}$. On the other hand, $\tau_{2}$ is about 100 ps below $50 \mathrm{~K}$ and decreases at higher temperatures. The temperature dependence of $\tau_{2}$ shows good agreement with that of the PL lifetime. It is noteworthy that the FEC signal intensity is strongly reduced at around $200 \mathrm{~K}$ because of the weak nonlinear response. At high temperatures, the upconverted PC exhibits sublinear power dependence while a superlinear power dependence appears below $200 \mathrm{~K} .^{28}$

The PL lifetime reflects that of the minority (short-lived) carriers in the radiative two-carrier (electron-hole) recombination process. We assume that $\tau_{2}$ represents the lifetime of photoexcited electrons in QWIs because the electron's effective mass is less than that of the hole. ${ }^{29}$ The thermal activation energy derived from the temperature dependence of the PL lifetime and $\tau_{2}$, which is $\sim 20 \mathrm{meV},{ }^{30}$ is consistent with the expected barrier height for electrons in QWIs, ${ }^{31,32}$ thereby suggesting that $\tau_{2}$ is determined by the thermal upconversion of electrons to the barrier at high temperatures above $60 \mathrm{~K}$, in which temperature region $\tau_{2}$ decreases with temperature. Consequently, $\tau_{1}$ is attributed to the hole lifetime in QWIs. Because of the very short electron lifetime and the relatively long hole lifetime at high temperatures, we consider that the photoexcited electron is extracted above the potential barrier immediately after the $\mathrm{e}-\mathrm{h}$ pair generation, leaving behind a photoexcited hole in the QWI. When a second $\mathrm{e}-\mathrm{h}$ pair is excited in the QWI, an electron-hole-hole state is realized, which leads to the carrier upconversion through the three-carrier AR process, as illustrated in Fig. 4(c).

Based on the above reasoning, we conclude that the room-temperature upconversion rate is dominated by the hole lifetime in InAs QWIs. The very small PL efficiency at room temperature indicates that the radiative recombination rate is negligibly small. We speculate that the long hole lifetime is due to it being trapped at nonradiative recombination centers in QWIs or its migration into deep InAs QDs through the WLs. ${ }^{33}$ The observed carrier lifetimes in QWIs are considerably smaller than the reported carrier lifetimes in QDs despite the relatively higher QWI upconversion efficiencies. $^{9,34,35}$ This indicates that QWIs exhibit a large AR coefficient, and thus, the upconversion efficiency can be improved by suppressing the nonradiative relaxation rate. This suppression can be achieved by fabricating high-quality InAs layers and ensuring the spatial separation of QWIs and QDs.

Finally, it is worth mentioning the potential application of QWIs into IBSCs. We have previously estimated the maximum conversion efficiency of the AlGaAs single junction SC $(28.2 \%)$ including ideal QWIs under 1-sun illumination as $35.7 \%$ under assumption of $100 \%$ AR upconversion efficiency in the QWI. ${ }^{9}$ However, an upconversion efficiency of only $2 \%$ is currently achieved in our samples for moderate continuous-wave excitation $\left(1.37 \mathrm{eV}, 0.5 \mathrm{~W} / \mathrm{cm}^{2}\right.$, corresponding to about 42 sun) at room temperature. ${ }^{9}$ Since the upconversion rate is dominated by the hole lifetime in QWIs, IBSCs based on the QWI concept will become practical after improvement of the hole lifetime by at least one order of magnitude.

In conclusion, we investigated the ultrafast carrier upconversion dynamics in InAs QWIs embedded in $\mathrm{Al}_{\mathrm{x}} \mathrm{Ga}_{1-\mathrm{x}} \mathrm{As}$ by PC-FEC. We estimated the hole lifetime at room temperature in a QWI to be $\sim 200 \mathrm{ps,} \mathrm{which} \mathrm{is} \mathrm{reduced}$ under high-density excitation due to the AR process. The large difference between the electron and hole lifetimes suggests that the three-body upconversion process (electron-hole-hole) plays a dominant role in carrier upconversion through the AR process at room temperature. Our results provide insights into the physics underlying the carrier dynamics in quantum nanostructures, which would enable us to deduce suitable design principles for efficient IBSCs utilizing carrier upconversion processes.

This work was supported by the Sumitomo Electric Industries Group CSR Foundation, JST-CREST, and KAKENHI (25247052).

\footnotetext{
${ }^{1}$ V. I. Klimov, A. A. Mikhailovsky, D. W. McBranch, C. A. Leatherdale, and M. G. Bawendi, Science 287, 1011 (2000).

${ }^{2}$ R. D. Schaller and V. I. Klimov, Phys. Rev. Lett. 92, 186601 (2004).
} 
${ }^{3}$ R. J. Ellingson, M. C. Beard, J. C. Johnson, P. R. Yu, O. I. Micic, A. J. Nozik, A. Shabaev, and Al. L. Efros, Nano Lett. 5, 865 (2005).

${ }^{4}$ A. Ueda, K. Matsuda, T. Tayagaki, and Y. Kanemitsu, Appl. Phys. Lett. 92, 233105 (2008).

${ }^{5}$ Y. Kanemitsu, Acc. Chem. Res. 46, 1358 (2013).

${ }^{6}$ J. T. Stewart, L. A. Padilha, W. K. Bae, W.-K. Koh, J. M. Pietryga, and V. I. Klimov, J. Phys. Chem. Lett. 4, 2061 (2013).

${ }^{7}$ A. Luque and A. Martí, Phys. Rev. Lett. 78, 5014 (1997).

${ }^{8}$ D. M. Tex and I. Kamiya, Phys. Rev. B 83, 081309(R) (2011).

${ }^{9}$ D. M. Tex, I. Kamiya, and Y. Kanemitsu, Phys. Rev. B 87, 245305 (2013).

${ }^{10}$ D. M. Tex, I. Kamiya, and Y. Kanemitsu, Sci. Rep. 4, 4125 (2014).

${ }^{11}$ A. Martí, E. Antolín, C. R. Stanley, C. D. Farmer, N. López, P. Díaz, E. Cánovas, P. G. Linares, and A. Luque, Phys. Rev. Lett. 97, 247701 (2006).

${ }^{12}$ Y. Okada, T. Morioka, K. Yoshida, R. Oshima, Y. Shoji, T. Inoue, and T. Kita, J. Appl. Phys. 109, 024301 (2011).

${ }^{13}$ K. Nishikawa, Y. Takeda, T. Motohiro, D. Sato, J. Ota, N. Miyashita, and Y. Okada, Appl. Phys. Lett. 100, 113105 (2012).

${ }^{14}$ Y. Yamada and Y. Kanemitsu, Appl. Phys. Lett. 101, 133907 (2012).

${ }^{15}$ Y. Yamada, T. Nakamura, M. Endo, A. Wakamiya, and Y. Kanemitsu, J. Am. Chem. Soc. 136, 11610 (2014).

${ }^{16}$ F. Rossi and T. Kuhn, Rev. Mod. Phys. 74, 895 (2002).

${ }^{17}$ J. Shah, Ultrafast Spectroscopy of Semiconductors and Semiconductor Nanostructures, Springer Series in Solid-State Sciences (Springer, New York, 1999).

${ }^{18}$ A. Solbrand, H. Lindstrom, H. Rensmo, A. Hagfeldt, S. E. Lindquist, and S. Sodergren, J. Phys. Chem. B 101, 2514 (1997).

${ }^{19}$ N. M. Gabor, Z. Zhong, K. Bosnick, and P. L. McEuen, Phys. Rev. Lett. 108, 087404 (2012)

${ }^{20}$ L. Q. Phuong, M. Okano, Y. Yamada, A. Nagaoka, K. Yoshino, and Y. Kanemitsu, Appl. Phys. Lett. 103, 191902 (2013).
${ }^{21}$ M. Freitag, T. Low, F. N. Xia, and P. Avouris, Nat. Photon. 7, 53 (2013).

${ }^{22}$ C. H. Lee, Picosecond Optoelectronic Devices (Academic Press, Inc., New York, 1984).

${ }^{23}$ L. Prechtel, L. Song, S. Manus, D. Schuh, W. Wegscheider, and A. W. Holleitner, Nano Lett. 11, 269 (2011).

${ }^{24}$ R. Heitz, T. R. Ramachandran, A. Kalburge, Q. Xie, I. Mukhametzhanov, P. Chen, and A. Madhukar, Phys. Rev. Lett. 78, 4071 (1997).

${ }^{25}$ See supplementary material at http://dx.doi.org/10.1063/1.4926569 for sample characteristics and FEC measurement principle.

${ }^{26}$ D. von der Linde and J. Kuhl, J. Lumin. 24-25, 675 (1981).

${ }^{27}$ Y. Miyauchi, K. Matsuda, and Y. Kanemitsu, Phys. Rev. B 80, 235433 (2009).

${ }^{28}$ D. M. Tex, T. Ihara, I. Kamiya, and Y. Kanemitsu, Jpn. J. Appl. Phys., Part 1 53, 05FV01 (2014).

${ }^{29}$ C. E. Pryor and M.-E. Pistol, Phys. Rev. B 72, 205311 (2005).

${ }^{30}$ The thermal activation energy for electrons was estimated using Arrhenius plots of $\tau_{2}$ and $\tau_{\mathrm{PL}}$.

${ }^{31}$ The QWI confinement energy $(\sim 230 \mathrm{meV})$ is the sum of the electron and hole barrier heights. Due to momentum conservation, the energy is differently distributed. Based on the electron and hole effective masses in InAs $\left(m_{e}{ }^{*}=0.026 m_{0}\right.$ and $\left.m_{h}{ }^{*}=0.04 m_{0}\right)$ according to Ref. 32, we can calculate the electron barrier height as $\sim 14 \mathrm{meV}$.

${ }^{32}$ M. Fox, Optical Properties of Solids (Oxford University Press, Inc., New York, 2010).

${ }^{33}$ W.-H. Chang, T. M. Hsu, C. C. Huang, S. L. Hsu, C. Y. Lai, N. T. Yeh, T. E. Nee, and J.-I. Chyi, Phys. Rev. B 62, 6959 (2000).

${ }^{34}$ D. Sato, J. Ota, K. Nishikawa, Y. Takeda, N. Miyashita, and Y. Okada, J. Appl. Phys. 112, 094305 (2012)

${ }^{35}$ T. E. J. Campbell-Ricketts, N. A. J. M. Kleemans, R. Nötzel, A. Yu. Silov, and P. M. Koenraad, Appl. Phys. Lett. 96, 033102 (2010). 